\title{
Atmospheric temperature dependence of muon intensity measured by the GRAPES-3 experiment
}

\author{
K. P. Arunbabu* ${ }^{* a b}$, S. Ahmad ${ }^{a, c}$, A. Chandra ${ }^{a, b, c}$, S.R. Dugad ${ }^{a, b}$, S.K. Gupta ${ }^{a, b}$, B. \\ Hariharan $^{a, b}$, Y. Hayashi ${ }^{a, d}$, P. Jagadeesan ${ }^{a, b}$, A. Jain ${ }^{a, b}$, V.B. Jhansi ${ }^{a, b}$, S. \\ Kawakami $^{a, d}$, H. Kojima ${ }^{a, e}$, P.K. Mohanty ${ }^{a, b}$, S.D. Morris ${ }^{a, b}$, P.K. Nayak ${ }^{a, b}$, A. \\ Oshima $^{a, f}$, B.S. Rao ${ }^{a, b}$, L.V. Reddy ${ }^{a, b}$, S. Shibata ${ }^{a, f}$, K. Tanaka ${ }^{a, g}$, M. Zuberi ${ }^{a, b}$ \\ ${ }^{a}$ GRAPES-3 Experiment, Cosmic Ray Laboratory, Raj Bhavan, Ooty 643001, India \\ ${ }^{b}$ Tata Institute of Fundamental Research, Mumbai 400005, India \\ ${ }^{c}$ Aligarh Muslim Univeristy, Aligarh 202002, India \\ ${ }^{d}$ Graduate School of Science, Osaka City University, Osaka 558-8585, Japan \\ ${ }^{e}$ Faculty of Engineering, Aichi Insitute of Technology, Toyota City, Aichi 470-0392, Japan \\ ${ }^{f}$ College of Engineering, Chubu University, Kasugai, Aichi 487-8501, Japan \\ ${ }^{g}$ Hiroshima City Univeristy, Asa-Minami-Ku, Hiroshima, 731-3194, Japan \\ E-mail: arun.babu@tifr.res.in
}

The large area $\left(560 \mathrm{~m}^{2}\right)$ GRAPES-3 tracking muon telescope located at Ooty, India has been operating uninterruptedly since 2001. Everyday it records $4 \times 10^{9}$ muons of energy $>1 \mathrm{GeV}$ with an angular resolution of $\sim 4^{\circ}$. Atmospheric temperature variation affects the rate of decay of these $\mathrm{GeV}$ muons produced by the galactic cosmic rays (GCRs), which in turn modulates the intensity of detected muons. Since the daily temperature induced variation combines with the diurnal modulation of the GCRs by the magnetized solar wind, it becomes rather difficult to segregate the respective contributions of these two phenomena. A small seasonal variation in the intensity of cosmic ray muon $(\sim 0.4 \%)$ with periodicity $\sim 1$ year $(\mathrm{Yr})$ was measured by analyzing the GRAPES-3 data of six years (2005-2010). The effective temperature ' $T_{\text {eff' }}$ ' of the upper atmosphere above Ooty also displayed a similar periodic variation with an amplitude of $\sim 1 \mathrm{~K}$, which was responsible for the observed seasonal variation in the muon intensity. At GeV energies, the muons detected by the GRAPES-3 show an anti-correlation with $\mathrm{T}_{\text {eff }}$ calculated by using a hadronic attenuation length $\lambda$. Using the fast Fourier transform (FFT) technique and making use of the anti-correlation between the seasonal variation of $\mathrm{T}_{\text {eff }}$ with the muon intensity, we calculated the temperature coefficient $\alpha_{\mathrm{T}}$. The magnitude of $\alpha_{\mathrm{T}}$ was found to scale with the assumed attenuation length $\lambda$, which we varied within a range of $80-180 \mathrm{gcm}^{-2}$. However, the actual magnitude of the correction was found to be independent of the value of $\lambda$.

Keywords: cosmic rays, muons, atmospheric temperature, fast Fourier transform

35th International Cosmic Ray Conference - ICRC2017

10-20 July, 2017

Bexco, Busan, Korea

${ }^{*}$ Speaker. 


\section{Introduction}

Galactic cosmic ray (GCR) intensity variations observed at ground-level has been studied for decades. These variations serve as an effective probe for the space-weather studies. The effects of CMEs in the near-Earth enviornment can be studied using cosmic rays [1, 2, 3, 4]. Solar modulations due to periodic effects such as the 27 day (d) solar rotation, 11 Yr solar cycle, and $22 \mathrm{Yr}$ solar magnetic cycle, result in similar periodic response in the observed GCR intensity [5, 6]. Earth's rotation in the ambient solar wind results in the detections of daily modulations of GCRs, known as solar diurnal anisotropy [7].

The GCRs interact with the molecules in the atmosphere and produce secondary particles, which are largely mesons like pions and kaons, which decays result in the production of muons of energies of several $\mathrm{GeV}$. These muon while traveling down in the atmosphere suffers energy losses (dominated by ionization) $\leq 2 \sec (\theta) \mathrm{GeV}$ before reaching the ground-level. A large fraction of muons survive down to ground-level with a median energy of $4 \mathrm{GeV}$.

The analysis of the ground-based muon flux is an effective tool for the space weather study and effect of CMEs on the magnetosphere of the Earth [8], however complex calculations and corrections for the atmospheric modulations should be performed. The ground-based detectors including the GRAPES-3 displays a variations in muon intensity observed correlated with the changes in the atmospheric pressure [9]. In addition to this muon intensity displays a seasonal variation correlated with the temperature variations in the upper atmosphere, this was first postulated in 1952 [10]. High energy muon observations display a positive temperature dependence [11, 12], where as low energy muon observations show a negative temperature dependence.

Because of its near-equatorial $\left(11.4^{\circ} \mathrm{N}\right)$ location, the seasonal variations observed in GRAPES3 is relatively small specially when compared to the variation seen in other experiments located at high latitudes. In the present work a we explain a unique methodology, used to measure the temperature dependence of the muon intensity by using the GRAPES-3 data of six years from 2005 to 2010 .

\section{The GRAPES-3 experiment}

The GRAPES-3 experiment is located at Ooty, India $\left(11.4^{\circ} \mathrm{N}\right.$ latitude, $76.7^{\circ} \mathrm{E}$ longitude, and $2200 \mathrm{~m}$ altitude). It consist of two main components, i) an air shower array of 400 scintillation detectors (each of $1 \mathrm{~m}^{2}$ ) arranged in a hexagonal geometry, with a distance of $8 \mathrm{~m}$ between adjacent detectors $[13,14]$, ii) a large area tracking muon telescope, which is a unique instrument used to search for high energy protons intensity during the active phase of a solar flare or a CME, which provides a high statistics, directional measurement of the muon flux [1, 2, 3, 7, 15, 16, 17]. This tracking muon telescope covers an area of $560 \mathrm{~m}^{2}$, consisting of a total of 16 modules, each $35 \mathrm{~m}^{2}$ in area and having an energy threshold of $\sec (\theta) \mathrm{GeV}$, for the muons arriving along a direction with zenith angle $\theta$. The observed muon rate of $\sim 3000 \mathrm{~s}^{-1}$ per module, yields a total muon rate $\sim 3 \times 10^{6} \mathrm{~min}^{-1}$ for the entire telescope [15]. This high-statistics, directional record of the muon intensity allows changes even at the level of $0.1 \%$ to be estimated accurately on a time scale of a minute. 
The basic element of this muon telescope is the proportional counter (PRC) with dimensions of $600 \mathrm{~cm} \times 10 \mathrm{~cm} \times 10 \mathrm{~cm}$. Muon modules are assemblies of PRCs placed in a four-layer configuration with alternate layers arranged in orthogonal directions. 4 adjacent modules located under common concrete shielding make a supermodule. An energy threshold of $\sec (\theta) \mathrm{GeV}$ for the muons in a direction of zenith angle ' $\theta$ ' is achieved by placing a $550 \mathrm{~g} \mathrm{~cm}^{-2}$ concrete blocks above the bottom most PRC layer.

\section{Seasonal variation in muon data}

In this analysis we used muon data from 16 modules, in every 3 hour interval for 6 years from 2005-2010. We have applied the necessary efficiency correction for the 16 modules to do precision studies, after which the measured muon intensities from these modules agreed within the statistical accuracy of the data $[18,19]$. Thereafter we performed pressure correction to remove the 12 hour periodic variations of muon intensity correlated with the atmospheric pressure $[9,18,19]$. The 3 hour averaging of the data resulted in a typical statistical error of $<0.01 \%$.

Atmospheric temperature data for different pressure levels were obtained from MDISC data subset[20]. Effective temperature ' $\mathrm{T}_{\text {eff' }}$ ' above Ooty were calculated using the temperature data over a range of latitude, and longitude covering the location of the GRAPES-3 site. An extensive Monte Carlo simulations were carried out to identify the muon production height of muons that are detected in GRAPES-3, which showed that the detected muons were produced at altitudes ranging from 6 to $30 \mathrm{~km}$ above the sea-level. In this study we considered the atmospheric temperature at 22 different pressure levels corresponds to these height. The small duration variation in upper atmosphere ( like daily or few days) is small compared with the seasonal variation. Temperature variation in different atmospheric layers are not uniform, hence we cannot predict the $\pi$ or $\mu$ production occurring entirely at one level. But the perturbation of intensity caused by variations of temperature are small, and we can treat them in terms of effective temperature $\left(T_{e f f}\right)$, weighted average of temperature from the surface to top of atmosphere [10]. We estimated the effective temperature of the atmosphere as,

$$
\mathrm{T}_{\text {eff }}=\frac{\sum \mathrm{e}^{\frac{-\mathrm{x}}{\lambda}} \mathrm{T}(\mathrm{x}) \Delta \mathrm{x}}{\sum \mathrm{e}^{\frac{-x}{\lambda}} \Delta \mathrm{x}}
$$

where ' $x$ ' is the atmospheric depth, in our case it is identified with the atmospheric pressure, $\lambda$ is the attenuation length of the hadrons, and $\mathrm{T}(\mathrm{x})$ is the temperature at a depth ' $\mathrm{x}$ '.

The variation of the muon rate $\left(R_{3 h}\right)$ is shown in Fig. 1a, and that of the $\mathrm{T}_{\text {eff }}$ in Fig. $1 \mathrm{~b}$, in three-hour intervals from 1 January 2005 to 31 December 2010 . The $\mathrm{T}_{\text {eff }}$ was calculated by using $\lambda=120 \mathrm{~g} \mathrm{~cm}^{-2}$. The $R_{3 h}$ shows variation due to the solar activity (Forbush decrease amplitudes of up to $3 \%$ ) along with the variation due to the seasonal change in the atmospheric temperature. The $R_{3 h}$, and $\mathrm{T}_{\text {eff }}$ displays a clear anti-correlation with a periodicity $1 \mathrm{Yr}$. For the low energy muons observed in GRAPES-3, an increase in the temperature causes expansion of the atmosphere resulting in greater probability for the decay of these muons, giving rise to the observed anticorrelation. The muon intensity variation $\frac{\Delta \mathrm{R}_{3 \mathrm{~h}}}{<\mathrm{R}_{3 \mathrm{~h}}>}$ is related to $\Delta \mathrm{T}$ as,

$$
\frac{\Delta \mathrm{R}_{3 \mathrm{~h}}}{<\mathrm{R}_{3 \mathrm{~h}}>} \times 100=\alpha_{\mathrm{T}} \Delta \mathrm{T}
$$




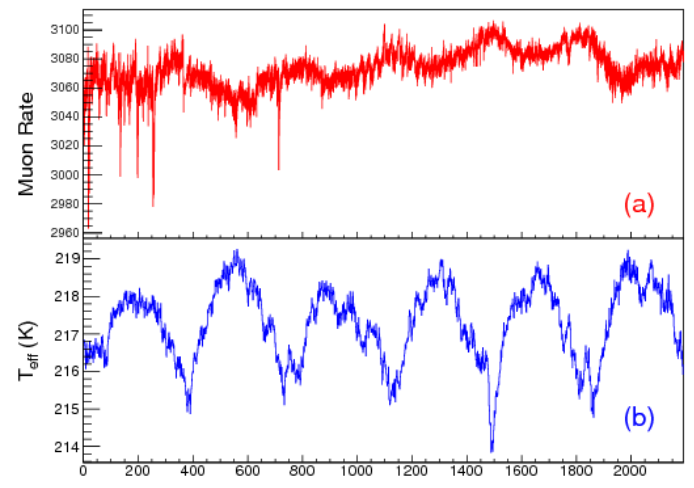

Figure 1: Variation of, (a) mean muon intensity, (b) $\mathrm{T}_{\text {eff }}$ during six years (2005-2010).

where $\alpha_{\mathrm{T}}$ is the temperature coefficient which is expressed in units of $\% \mathrm{~K}^{-1}$.

\section{Temperature coefficient $\alpha_{\mathrm{T}}$ by conventional method}

In this method we first removed the small variations in the $R_{3 h}$ due to solar phenomena like FDs and 27 day periodicities, for this we applied a running average filter with a window width of 60 days. The resultant muon intensity(\%), and $\mathrm{T}_{\text {eff }}$ are shown in Fig. 2 in the top, and bottom panels, respectively.

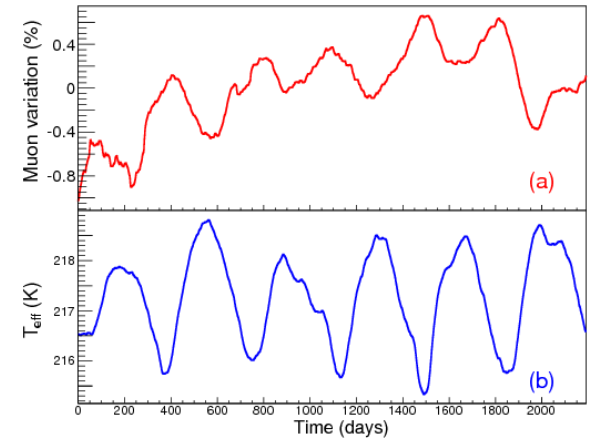

Figure 2: Variation of, (a) muon intensity, (b) $\mathrm{T}_{\text {eff }}$ (K) by using $60 \mathrm{~d}$ running average.

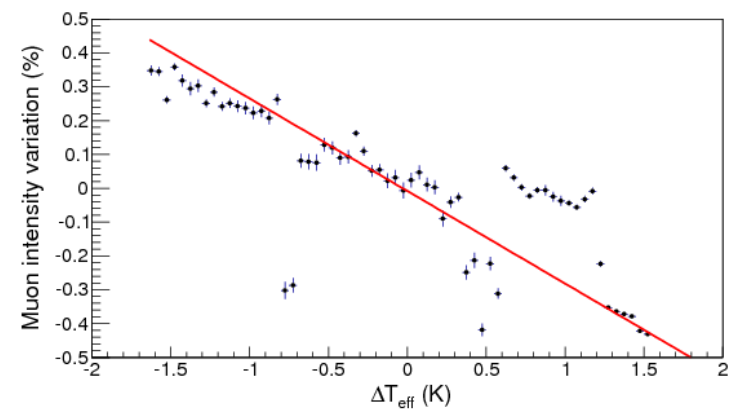

Figure 3: Dependence of muon intensity on $\Delta \mathrm{T}$ (K) for the data of 2005-2010. Linear fit yields a slope of $-0.27 \% \mathrm{~K}^{-1}$, and $\chi^{2}$ of 151 per DOF

The dependence of the $R_{3 h}$ on $\mathrm{T}_{\text {eff }}$ is shown in Fig. 3. Each point in Fig. 3 represents the mean muon intensity (\%) within a temperature range of $0.05 \mathrm{~K}$. The vertical error bar at each point represents the root mean square spread in the muon intensity. The modulation of the GCR intensity due to various solar phenomena have made this dependence rather complex. A linear fit to this data is shown in Fig. 3 by the solid line, which is a poor fit with a large $\chi^{2}=151$ per degree of freedom (DOF). The temperature coefficient from this fit is $\alpha_{\mathrm{T}}=-0.27 \% \mathrm{~K}^{-1}$. One can think of choosing data sets of periods of low solar activity, but practically it is nearly impossible to collect these data sets within a relatively constant solar wind parameters. In addition, the GCR intensity displays correlation with the interplanetary magnetic field (IMF) with a delay during the FD events [2], this delay in response of the GCR intensity to the IMF may present even during the solar quiet periods. Thus, a reasonably accurate estimation of the $\alpha_{\mathrm{T}}$ is not feasible by the conventional method. 


\section{Temperature coefficient $\alpha_{\mathrm{T}}$ by FFT method}

The atmospheric temperature displays a clear variation with a period of 1 Yr as seen in Fig. $1 \mathrm{~b}$. This variation produces a corresponding response in the muon intensity detected at the GRAPES-3 as seen in Fig. 1a. To quantitatively study this correlation, fast Fourier transform (FFT) of both these data sets were obtained. The FFT of the $R_{3 h}$ and $\mathrm{T}_{\text {eff }}$ data were obtained by using a standard routine TVirtualFFT built in the ROOT framework [21], which also incorporated an FFT library FFTW (Fastest Fourier Transform in the West) [22].

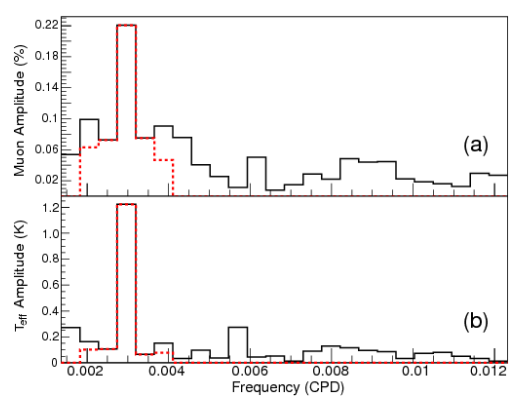

Figure 4: FFT spectrum for six years of data, (a) muon intensity, (b) $\mathrm{T}_{\text {eff }}$. Filtered spectra shown by dashed lines.

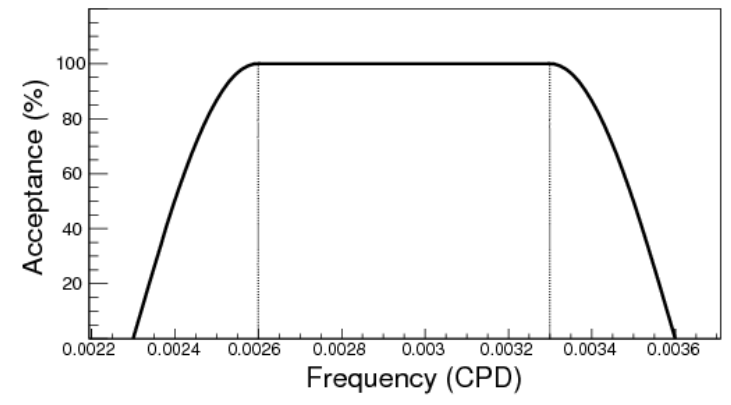

Figure 5: Filter centered at 0.00295 CPD for muon intensity, and $\mathrm{T}_{\text {eff }}$ data. $100 \%$ acceptance enclosed by vertical dashed lines.

The FFT amplitude for the $R_{3 h}$ and $\mathrm{T}_{\text {eff }}$ are shown by solid lines in Fig. 4a,b, respectively. The muon amplitude shows a peak at 1/365 CPD (1 Yr), and the width of the peak covers the frequency range from 0.0027 to $0.0032 \mathrm{CPD}$. The amplitude for $\mathrm{T}_{\text {eff }}$ also displays a dominant peak at the same period, clearly showing the contribution of the atmospheric temperature on the seasonal variation seen in the muon intensity. This periodicity was exploited to eliminate the non-atmospheric effects of solar origin from the muon data with the objective of obtaining a more accurate estimate of $\alpha_{\mathrm{T}}$. For this purpose a narrow-band filter $\mathrm{W}(\mathrm{f})$ was devised to select frequencies centered at $0.00295 \mathrm{CPD}$ as described below,

$$
\mathrm{W}(\mathrm{f})=\left\{\begin{array}{l}
1, \quad \text { if }\left|\mathrm{f}-\mathrm{f}_{\mathrm{c}}\right| \leq \Delta \mathrm{f} \\
\sin \frac{\pi}{2} \frac{\left|\mathrm{f}-\mathrm{f}_{\mathrm{c}}\right|}{\Delta \mathrm{f}} \text { if } \Delta \mathrm{f}<\left|\mathrm{f}-\mathrm{f}_{\mathrm{c}}\right| \leq 2 \Delta \mathrm{f} \\
0, \quad \text { if }\left|\mathrm{f}-\mathrm{f}_{\mathrm{c}}\right|>2 \Delta \mathrm{f}
\end{array}\right.
$$

For this analysis a filter was constructed with central frequency, $\mathrm{f}_{\mathrm{c}}=0.00295 \mathrm{CPD}, \Delta \mathrm{f}=0.00035 \mathrm{CPD}$ as shown in Fig. 5. In the frequency range from 0.0026 to $0.0033 \mathrm{CPD}$, the filter had an acceptance of $100 \%$. Thereafter, the filter acceptance smoothly decreased to zero with a sinusoidal shape in the range $0.0026-0.0023 \mathrm{CPD}$, as well as $0.0033-0.0036 \mathrm{CPD}$, respectively. The filter acceptance was zero outside the range listed above.

The frequency spectrum of the muon, and $T_{\text {eff }}$ data were subjected to this narrow-band filter, and the resultant spectrum for the muon data is shown by the dashed line in figure $4 \mathrm{a}$. As expected, the filtered spectrum shows a smooth transition on either side of the peak, while removing the frequency component below 0.0023 , and above $0.0036 \mathrm{cpd}$. An identical exercise was repeated for the $T_{\text {eff }}$ data, and the corresponding spectra are shown in figure $4 \mathrm{~b}$. 
A large fraction of the amplitude in the spectrum at $0.00295 \mathrm{CPD}$ was contained in a single bin as may be seen from Fig. 4. Yet there was a significant spillover into the neighboring bins. Such spillover can easily occur in case the duration of the data set was not a multiple of the underlying period, or if the data were not continuous [23]. But, in the present case due care was taken to ensure that these situations did not occur.

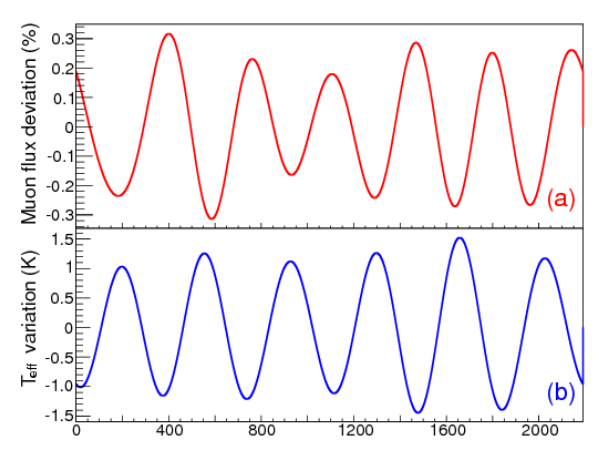

Figure 6: IFFT data in time domain for, (a) muon intensity (\%), (b) $\mathrm{T}_{\text {eff }}(\mathrm{K})$.

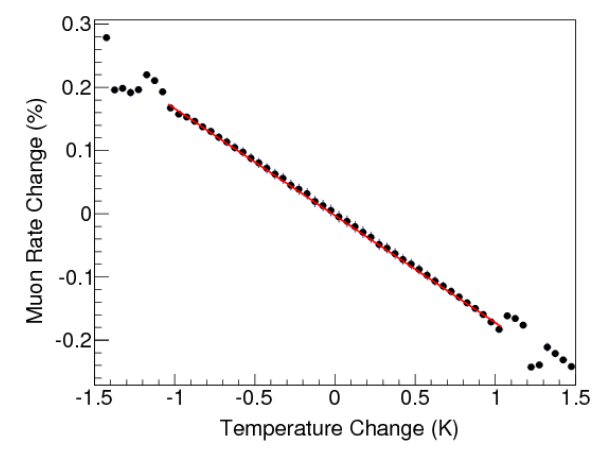

Figure 7: Variation in muon intensity as a function of $\Delta \mathrm{T}$ relative to respective mean values.

An inverse fast Fourier transform (IFFT) was performed on the filtered frequency spectra to convert them back into the time domain. The resultant IFFT data for the muons are shown in Fig. 6a, and for for the effective temperature ' $\mathrm{T}_{\mathrm{eff}}$ ' in Fig. 6b. The near-perfect anti-correlation of these two data sets demonstrates the 1 Yr periodic contribution of the $T_{\text {eff }}$ on the muon intensity.

After the IFFT, the muon data are plotted against $\Delta \mathrm{T}$ as shown in Fig. 7. Here, each data point represents the variation in the muon intensity for $0.05 \mathrm{~K}$ change in $\Delta \mathrm{T}$. The error bars corresponding to each data point were also plotted which are invisible since their sizes are smaller than the size of the symbols used. A linear fit to the data is shown in Fig. 7 by solid line. The slope of the fit representing the temperature coefficient $\alpha_{\mathrm{T}}$ was found to be $-0.17 \% \mathrm{~K}^{-1}$. The fit yielded an extremely small statistical error primarily because of the high-statistics of GRAPES-3 data. However, in the present work the systematic effects on the muon intensity due to other phenomena can not be entirely ruled out due to the use of only six cycles (from six years) of data.

As explained in $\S 3$ the value of the temperature coefficient $\alpha_{\mathrm{T}}=-0.17 \% \mathrm{~K}^{-1}$ was obtained for $\lambda=120 \mathrm{~g} \mathrm{~cm}^{-2}$. Subsequently, $\alpha_{\mathrm{T}}$ was calculated for a wide range of $\lambda$ ranging $80-180$ in steps of $20 \mathrm{~g} \mathrm{~cm}^{-2}$. The first and second column in Table 1 lists $\lambda$ and $\alpha_{\mathrm{T}}$, the third column the mean amplitude of the seasonal temperature variation $\Delta \mathrm{T}$, and the last column is the product of $\alpha_{\mathrm{T}}$, and $\Delta \mathrm{T}$ which represents the $6 \mathrm{Yr}$ mean seasonal amplitude of the muon intensity variation. The $\alpha_{\mathrm{T}}$ values listed in Table 1 display a linear dependence on $\lambda$ as shown in Fig. 8 , the seasonal amplitude of the muon intensity variation $\alpha_{\mathrm{T}} \times \Delta \mathrm{T}$ is also plotted in Fig. 8 as a function of $\lambda$ which is shown on the secondary Y-axis. It is important to note that Fig. 8 clearly shows that the seasonal amplitude of the muon intensity variation is almost independent of the assumed attenuation length $\lambda$.

\section{Summary}

Correction of the muon intensity for the changes in the atmospheric temperature is an essential requirement for the study of the long-term GCR intensity modulations of solar origin. The 


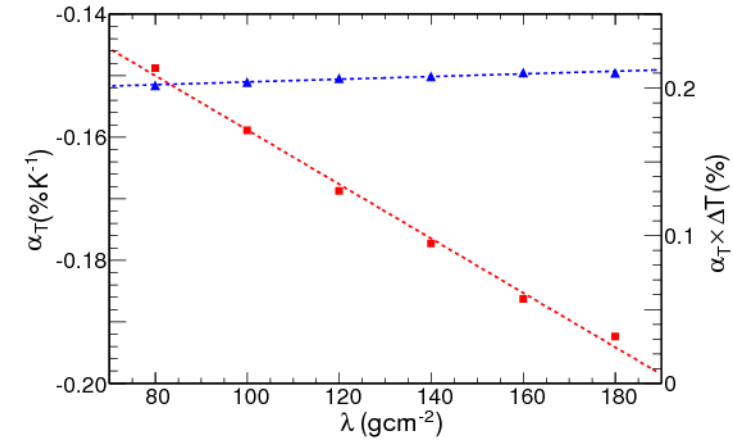

Figure 8: Variation of $\alpha_{\mathrm{T}}$ as a function of $\lambda$ shown as filled squares. Muon intensity amplitude $\Delta \mathrm{I}_{\mu}$ $\left(\alpha_{\mathrm{T}} \times \Delta \mathrm{T}\right)$ variation in $\%$ shown as filled triangles.

\begin{tabular}{|c|c|c|c|}
\hline $\begin{array}{c}\lambda \\
\left(\mathrm{g} \mathrm{cm}^{-2}\right)\end{array}$ & $\begin{array}{c}\alpha_{\mathrm{T}} \\
\left(\% \mathrm{~K}^{-1}\right)\end{array}$ & $\begin{array}{c}\Delta \mathrm{T} \\
(\mathrm{K})\end{array}$ & $\alpha_{\mathrm{T}} \times \Delta \mathrm{T}$ \\
\hline 80 & -0.15 & 1.36 & $0.20 \%$ \\
\hline 100 & -0.16 & 1.28 & $0.20 \%$ \\
\hline 120 & -0.17 & 1.22 & $0.21 \%$ \\
\hline 140 & -0.18 & 1.17 & $0.21 \%$ \\
\hline 160 & -0.19 & 1.13 & $0.21 \%$ \\
\hline 180 & -0.19 & 1.09 & $0.21 \%$ \\
\hline
\end{tabular}

Table 1: Dependence of $\alpha_{\mathrm{T}}$ on attenuation length $\lambda$

high redundancy of the GRAPES-3 muon telescope allowed an almost uninterrupted stream of data for the years from 2005-2010, which made it possible to explore the atmospheric temperature dependence of the muon rate in detail. It is well-known that the atmospheric temperature, and the muon intensity are anti-correlated, but the interference of the solar origin modulations such as diurnal anisotropy, Forbush decrease events, $27 \mathrm{~d}$ solar rotation, and $11 \mathrm{Yr}$ periodicity complicate this relationship. A visual inspection of the time series $R_{3 h}$ for the 6 years $(2005-2010)$ revealed complex variations, which includes solar modulations along with the seasonal variations. Amplitude of seasonal variations variations seems to be very small compared to solar modulations. Thus, the presence of solar induced variations had made the study of the dependence of muon intensity on the atmospheric temperature rather challenging in the past.

Extensive Monte Carlo simulations using CORSIKA showed that the heights of production of muons detected in GRAPES-3 varied from 6 to $30 \mathrm{~km}$ above the mean sea level. Based on this atmospheric temperature data at 22 different pressure levels ranging from 10 to $775 \mathrm{hPa}$ were used for estimating the $\left(\mathrm{T}_{\text {eff }}\right)$. The muon intensity showed an anti-correlation with $\mathrm{T}_{\text {eff }}$, but the influence of the solar origin variations were more prominent. Hence it was important to remove the Sun induced variations for an accurate measurement of the temperature coefficient $\alpha_{\mathrm{T}}$. The $\alpha_{\mathrm{T}}$ from conventional method would contain large errors, was clear from the poor correlation of the muon intensity, and $\mathrm{T}_{\text {eff }}$, and a large value of $\chi^{2}$ in the fit shown in Fig. 3. Thus, it became obvious that the non-atmospheric variations needed to be effectively eliminated before calculating $\alpha_{\mathrm{T}}$. The shortterm variations were removed by using the conventional method, but the slow variations caused by the solar effects still survived.

The seasonal variations of $\mathrm{T}_{\text {eff }}$ displayed a dominant $1 \mathrm{Yr}$ period with an amplitude of $\sim 1 \mathrm{~K}$. This produced a corresponding variation in the muon intensity observed in the GRAPES-3 data. The FFT spectrum of $\mathrm{T}_{\text {eff }}$ and muon intensity showed a dominant $1 \mathrm{Yr}$ component, which was used to eliminate the contributions of solar origin in the muon data by the application of a narrow-band filter centered at $1 \mathrm{Yr}$, in both the muon, and $\mathrm{T}_{\text {eff }}$ data sets. The filtered muon, and $\Delta \mathrm{T}$ data showed a strong anti-correlation as seen from Fig. 7 , which helped in a fairly reliable estimation of the temperature coefficient $\alpha_{\mathrm{T}}=-0.17 \% \mathrm{~K}^{-1}$. As discussed in $\S 5$ the magnitude of $\alpha_{\mathrm{T}}$ was found to be proportional to the assumed value of $\lambda$. However, the magnitude of the temperature deviation $\Delta \mathrm{T}$ was found to be inversely proportional to $\alpha_{\mathrm{T}}$ as shown in Table1. Thus the correction to the 
muon intensity $\Delta \mathrm{I}_{\mu}$ which is the product of $\alpha_{\mathrm{T}}$ and $\Delta \mathrm{T}$ was independent of the value of $\lambda$. This method yielded a robust correction to the muon intensity variation due to temperature effect which was independent of the assumed value of $\lambda$. After obtaining $\alpha_{\mathrm{T}}$, a correction in the muon intensity to remove the seasonal variations can be performed which should provide reliable data for further study of the long-term GCR variations of the solar origin.

\section{Acknowledgments}

We are grateful to D.B. Arjunan, G.P. Francis, V. Jeyakumar, S. Kingston, K. Manjunath, S. Murugapandian, B. Rajesh, K. Ramadass, C. Ravindran, V. Santoshkumar, M.S. Shareef, C. Shobana, R. Sureshkumar for their role in the efficient running of the experiment. We acknowledge the Goddard Earth Science Data, and the Information Services Center for making the temperature data available online.

\section{References}

[1] K.P. Arunbabu et al., Astron. Astrophys. 555 (2013) A139.

[2] K.P. Arunbabu et al., Astron. Astrophys. 580 (2015) A41.

[3] P. Subramanian et al., Astron. Astrophys. 494 (2009) 1107.

[4] K. Kudela et al., Space Sci. Rev. 93 (2000) 153.

[5] S.E. Forbush, J. Geophys. Res. 59 (1954) 525.

[6] D. Venkatesan, Badruddin Space Sci. Rev. 52 (1990) 121.

[7] P.K. Mohanty et al., Pramana J. Phys. 81 (2013) 343.

[8] P.K. Mohanty et al., Phys. Rev. Lett. 117 (2016) 171101.

[9] P.K. Mohanty et al., Astropart. Phys. 79 (2016) 23.

[10] P. Barrett et al., Rev. Mod. Phys. 24 (1952) 133.

[11] P. Desiati et al., Proc. of 32nd Int. Cosmic Ray Conf. Beijing (2011).

[12] P. Adamson et al., Phys. Rev. D 90 (2014) 012010.

[13] S.K. Gupta et al., Nucl. Instrum. Methods A 540 (2005) 311.

[14] S.K. Gupta et al., Nucl. Phys. B Proc. Suppl. 196 (2009) 153.

[15] T. Nonaka et al., Phys. Rev. D 74 (2006) 052003.

[16] H. Kojima, et al., Astropart. Phys. 62 (2015) 21.

[17] H. Kojima et al., Phys. Rev. D 91 (2015) 121303(R).

[18] S.K. Gupta, EPJ Web Conf. 52 (2013) 04005.

[19] P.K. Mohanty, Ph.D. Thesis, TIFR unpublished, (2014).

[20] http://disc.sci.gsfc.nasa.gov/daac-bin/FTPSubset.pl

[21] http://root.cern.ch/root/html/TVirtualFFT.html

[22] http://www.fftw.org

[23] F.J. Harris, Proc. IEEE 66 (1978) 51. 